ARTIGO DE REVIS ÃO

\section{ACUPUNTURA NAS ONDAS DE CALOR}

\section{ACUPUNCTURE ON HOT FLASHES}

\section{RESUMO}

Objetivo: Descrever as técnicas de acupuntura aplicadas para alívio de ondas de calor decorrentes do período do climatério. Método: Revisão sistemática na base de dados Medline, pelo método P.I.C.O, onde $\mathrm{P}=$ Postmenopausal, $\mathrm{I}=$ Acupuncture e $\mathrm{O}=$ Hot Flashes. Foram selecionados artigos científicos controlados e randomizados sobre o tema em questão. Um total de 12 artigos preencheu os critérios de inclusão; mulheres saudáveis na fase pré e pós-menopausa e mulheres com risco para utilização de terapia hormonal, relatando ondas de calor, submetidas às técnicas de acupuntura sistêmica e eletroacupuntura. Resultados: Estudos analisados indicam alívio dos sintomas para ondas de calor nas variantes severidade (média 50\%) e frequência (média 45\%), porém a eletroacupuntura, quando observada em longo prazo, apresenta melhor resultado em relação à acupuntura sistêmica. Conclusões: Estudos sugerem que a prática da acupuntura é segura e eficaz para amenizar ondas de calor, tanto em mulheres saudáveis como em mulheres com contra indicação para a reposição hormonal.

\section{PALAVRAS-CHAVE}

\section{Menopausa}

Pós-menopausa

Acupuntura

Ondas de calor
CADERNOS DE

NATUROLOGIA f) Unisul

Mariana Parro Lima

- Bacharel em Naturologia pela UAM. Mestre em Educação pela UNICAMP.

Mirian Ilda da Silva

- Fisioterapeuta Acupunturista

e Especialista em Saúde da

Mulher no Climatério (Faculdade de Saúde Pública - USP

Audrea Doglio Foletto

- Educadora Física e Especialista em

Saúde da Mulher no Climatério

(Faculdade de Saúde Pública - USP)

CORRESPON DÊNCIA:

Universidade Estadual de Campinas, Faculdade de Educação.

Av. Bertrand Russel, 801 DELART -

Laboratório OLHO

Cidade Universitária -13083-865 -

Campinas, SP - Brasil

Telefone: (19) 35215578

Recebido: 04/04/2013

Revisado: 11/05/2013

Aprovado: 13/06/2013 


\begin{abstract}
Objective: To describe the techniques of acupuncture applied on the relief of hot flashes in climacteric women. Method: Systematic review at Medline database, using the PICO method, where P = Postmenopausal, $\mathrm{I}=\mathrm{O}=$ Acupuncture and Hot Flashes, scientific articles and controlled trials on the topic in question. A total of 12 articles met the inclusion criteria, healthy women in the pre and post menopause and women at risk for hormone therapy, reporting hot flashes, submitted to the techniques of acupuncture and electro acupuncture systemic. Results: Studies analyzed indicate symptom relief for hot flashes in variants severity (mean 50\%) and frequency (average 45\%), but electro acupuncture, when viewed in the long run, has a better outcome for systemic acupuncture. Conclusions: Studies suggests that the practice of acupuncture is safe and effective to alleviate hot flashes in both healthy women and women with contraindications to hormone therapy.
\end{abstract}

KEY WORDS: Menopause. Postmenopausal. Acupuncture. Heat waves.

\section{INTRODUÇÃO}

Novas atitudes em relação à saúde e qualidade de vida vêm sendo observadas nos últimos anos, havendo, assim, uma crescente consciência em favor de uma medicina mais preventiva e de tratamentos alternativos, que são menos agressivos ao organismo. Dentre esses, estão incluídos os tratamentos para sintomas primários do climatério, principalmente quando se referem a fogachos, dores musculares, distúrbios do sono e fadiga ${ }^{19}$.

O climatério compreende o período em que ocorre a transição da fase reprodutiva para a fase não reprodutiva da vida da mulher. Inicia-se com o declínio da função ovariana e o anúncio da menopausa, essa denota a cessação permanente da menstruação, ou seja, o último fluxo menstrual, comprovado por meio da amenorreia espontânea por 12 meses consecutivos ${ }^{1}$. Esse período vem acompanhado de vários sintomas ${ }^{20}$.

Estudos demonstram que os fogachos (ondas de calor) são os desconfortos que mais incomodam essas mulheres, em torno de $75 \%{ }^{20}$. Kroemberg estima um valor ainda maior; $87 \% \%^{17-18}$.

Esses afrontamentos começam, tipicamente, um a dois anos antes da menopausa e podem persistir por um pouco mais de seis meses ou até por mais de 10 anos $^{23}$.

Deve-se considerar o climatério como um processo natural da mulher, onde a infertilidade determina o início de uma nova fase ${ }^{12}$, mas também observar que essa etapa da vida vem acompanhada de modificações que podem acelerar o processo do envelhecimento, tornando-a mais susceptível a alterações vasomotoras, atrofias e instabilidades psicoemocionais, aumentando o risco para doenças cardiovasculares (DCV) e osteoporose ${ }^{2}$ (1994).

Esses episódios ocorrem em torno dos 50 anos de idade ${ }^{2,12,18,23}$. À medida que os ovários envelhecem diminuem os ciclos menstruais, as ovulações são menos frequentes e cai a produção dos hormônios estrogênio e progesterona, causando irregularidade menstrual ${ }^{12}$.

Embora a menopausa esteja associada a mudanças na atividade central de opióides ao nível do eixo hipotálamo-hipófise-ovário (HPOA), que regulam o ciclo menstrual, esta não é um evento central e sim decorrente de insuficiência ovariana primária ${ }^{5,28}$.

Como o eixo HPOA permanece intacto durante a transição menopausal, o aumento do hormônio folículo estimulante (FSH) é uma resposta ao fracasso ou ausência dos hormônios ovarianos (Estradiol - E2), e a atresia do aparelho folicular resulta na diminuição da produção do estrogênio, levando a redução dos seus níveis e elevação do $\mathrm{FSH}^{26}$.

A reposição hormonal é o tratamento clínico mais comumente prescrito para as mulheres que relatam alguns sintomas da menopausa ${ }^{26}$, porém esse tratamento não é recomendado para muitas delas, por uma variedade de razões provenientes de absolutas contraindicações e reclamações individuais associadas com o tratamento ${ }^{11,24}$. 
Pesquisadores brasileiros consideram que a abordagem da mulher no climatério deveria ser ampliada e sugerem que os desconfortos poderiam ser abordados de diferentes maneiras e não simplesmente com a hormonioterapia. Contudo, as pesquisas de profissionais da saúde que abordam as mulheres climatéricas de forma holística são, numericamente, tímidas ou pouco divulgadas, o que reflete na carência de assistência oferecida a essas mulheres ${ }^{6}$.

Nos últimos anos, a busca por terapias complementares aumentou muito. Esta nova realidade impulsionou autoridades governamentais, sistemas, instituições e escolas de saúde, além dos profissionais da área, a adequarem-se a esta nova realidade.

$\mathrm{Na}$ Suécia, o aumento da incidência de câncer de mama nos últimos anos levou mulheres a procurarem tratamento não hormonal para os sintomas da menopausa ${ }^{14}$.

A Organização Mundial da Saúde preconiza em seu documento sobre Estratégias da Medicina Tradicional de 2002-2005 o desenvolvimento de estudos científicos para melhorar o conhecimento sobre as terapias complementares, bem como a segurança, eficácia e qualidade destas práticas ${ }^{27}$. Entre essas, a acupuntura tem demonstrado ser um dos tratamentos alternativos de maior aceitação em termos de efetividade.

A acupuntura faz parte de um conjunto de técnicas terapêuticas, que tem como base os preceitos da Medicina Tradicional Chinesa (MTC). É, atualmente, uma das terapias orientais mais populares em diversos países e tem sido amplamente utilizada na assistência à saúde, tanto nos aspectos preventivos quanto curativos.

Nas últimas décadas, a acupuntura vem se difundindo em função da crescente aceitabilidade pelas comunidades científicas. Estudos realizados sob uma perspectiva ocidental, envolvendo novos métodos de pesquisas, têm colaborado para esse fim ${ }^{27}$.

A acupuntura em ginecologia e obstetrícia é uma prática considerada recente ${ }^{4}$, um dos trabalhos mais antigos encontrados (datado de 1960), especificamente para transtornos da mulher, faz citação sobre a aplicação da acupuntura com eletroestimulação para curar pacientes com transtornos de anovulação (esterilidade) ${ }^{8}$.

Atualmente, essa especialidade tem trabalhado no sentido de evidenciar os fatos clínicos e promover maior bem estar para os transtornos de patologias exclusivamente femininos, dentre elas sintomas do climatério.

Desta forma, o objetivo desse trabalho foi identificar quais as técnicas de acupuntura são aplicadas para alívio da frequência e severidade das ondas de calor nas mulheres no climatério e sua efetividade.

\section{MÉTODOS}

Procedeu-se uma busca sistemática, na base de dados Medline, através do método P.I.C.O, onde P corresponde à população ou problema, I corresponde à intervenção ou procedimento, $C$ corresponde à comparação ou controle e O corresponde a Outcomes ou Desfechos. As palavras chaves correspondentes foram: $\mathrm{P}=$ Postmenopausal, $\mathrm{I}=$ Acupuncture e $\mathrm{O}=$ Hot Flashes.

Foram incluídos artigos de ensaio clínico randomizado e controlados realizados em mulheres saudáveis na fase pré e pós-menopausa e mulheres com risco para utilização de terapia hormonal, relatando ondas de calor, submetidas às técnicas de acupuntura sistêmica e eletroacupuntura. Encontramos 12 artigos com essas características, referindo-se ao tema do estudo. A partir daí, todos os textos foram traduzidos para o português, analisados quanto à população observada, critério de inclusão e exclusão semelhantes, as técnicas aplicadas, características das sessões.

Dos doze trabalhos analisados: quatro são da Suécia ${ }^{13,21,29,30}$, cinco dos USA ${ }^{3,10,15,22,25}$, um da Inglaterra $^{9}$, um da Noruega ${ }^{7}$ e um da China ${ }^{16}$.

Três desses trabalhos tem como objetivo avaliar se a acupuntura/eletroacupuntura é efetiva para os sintomas vasomotores em mulheres com história de câncer de mama ${ }^{10,13,21}$. Oito trabalhos têm como objetivo avaliar o efeito da acupuntura nos sintomas vasomotores em mulheres sem patologias prévias ${ }^{3,79,15,22,25,29-30}$.

Um dos trabalhos analisados tem como objetivo avaliar o efeito da acupuntura nos sintomas vasomo- 
tores em mulheres ovariectomizadas bilateralmen$\mathrm{te}^{\mathrm{e}}{ }^{16}$. Apesar das diferentes mulheres analisadas, todas possuíam o mesmo ponto em comum; ondas de calor sejam por menopausa espontânea ou induzida.

As técnicas de acupuntura observadas nos estudos analisados foram acupuntura sistêmica, tanto acupuntura tradicional chinesa como acupuntura sham (simulação), eletroacupuntura (EA) isolada e eletroacupuntura (EA) associada à auriculoterapia (acupressão), há citações de comparações com terapia de reposição hormonal (TRH) via oral, transdérmico ou placebo, relaxamento aplicado e cuidados habituais.

Dessa análise, citamos os aspectos mais relevantes dos trabalhos encontrados, em relação à aplicabilidade da acupuntura; tempo de tratamento ficou entre 8 e 13 semanas, totalizando 8 a 16 sessões. As sessões semanais variaram entre 1 e 2 sessões de 20 a 40 minutos cada uma. Os métodos observados foram: acupuntura sistêmica de acordo com a Medicina Tradicional Chinesa (MTC), acupuntura sistêmica de acordo com pontos específicos para sintomas da menopausa, acupuntura associada à auriculoterapia ou acupressão, eletroacupuntura com estímulo de $2 \mathrm{~Hz}$ e acupuntura-sham. Tivemos como métodos comparativos TRH (estrógeno oral ou transdérmico), cuidados habituais e relaxamento aplicado.

Os pontos mais aplicados foram: $\mathrm{VC} 4, \mathrm{BP} 6, \mathrm{~B} 23$, CS6, R7, B15, C7, VB20, VG20, R3. Dentro da MTC existe uma terminologia própria para caracterizar os pontos de acupuntura, explicaremos abaixo a função energética dos pontos mais relevantes encontrados nos trabalhos analisados (Quadro 1).

Quadro 1. Funções energéticas dos pontos mais aplicados

\begin{tabular}{l|l|l|l}
\hline Pontos & $\begin{array}{l}\text { Nome de acordo } \\
\text { com o Meridiano }\end{array}$ & Nome na M.T.C. & Explicação segundo a função energética \\
\hline VC4 & Vaso Concepção & Guanyuan & $\begin{array}{l}\text { Regulariza o Chong Mai e Ren Mai, } \\
\text { regulariza Qi e harmoniza o Xue }\end{array}$ \\
\hline BP*6 & Baço-Pancrêas & Sanyinjiao & $\begin{array}{l}\text { Regulariza e harmoniza o Qi dos 3 } \\
\text { meridianos da perna(tonifica BP, avantaja } \\
\text { e alimenta o Yin do Fígado e Rim). }\end{array}$ \\
\hline B23 & Bexiga & Shenshu & $\begin{array}{l}\text { Avantaja o Rim, a água faz recuar o calor } \\
\text { ao vazio do Yin, refresca os Rins }\end{array}$ \\
\hline R7 & Circulação Sexo ou & Neiguan & $\begin{array}{l}\text { Refresca o calor, apazigua a mente, } \\
\text { dilata o peito, refresca Yin e Xue }\end{array}$ \\
\hline B15 & Bexiga & Fuliu & $\begin{array}{l}\text { Tonifica Yang do Rim, atua na transpiração } \\
\text { Alimenta o sangue, apazigua a } \\
\text { mente, refresca o coração }\end{array}$ \\
\hline VB & Coração & ShenMen & $\begin{array}{l}\text { Refresca o coração, acalma a } \\
\text { mente, harmoniza o Yin }\end{array}$ \\
\hline VG20 & Vesícula Biliar & Fenghi & $\begin{array}{l}\text { Utilizado para perturbações da menopausa } \\
\text { para diminuir ondas de calor }\end{array}$ \\
\hline
\end{tabular}

Fonte: B. Aureroche(17) 


\section{RESULTADOS}

Os estudos revisados demonstram eficácia significativa da maioria das técnicas aplicadas, tanto nos grupos controle como nos grupos experimentais, sendo que a TRH é a mais eficaz para diminuir as frequências e severidades das ondas de calor, seguidas pela eletroacupuntura e acupuntura sistêmica.

Observamos ainda que, três desses estudos também avaliaram o efeito das técnicas nos sintomas da menopausa pelo índice de Kupperman e Blatt ${ }^{13,29-30}$, em outro estudo avaliou esses mesmos efeitos pelo Women's Health Questionnaire (WHQ) ${ }^{10}$.

A TRH alcançou uma média percentual de decréscimo para os sintomas da menopausa, segundo índice de Kupperman de 71,37\%, seguido pela eletroacupuntura; $52,85 \%$ e a acupuntura sistêmica; 45,95\% (Ilustrado no Gráfico 1).
Gráfico 1. Comparação das técnicas para sintomas da Menopausa segundo índice de Kupperman.

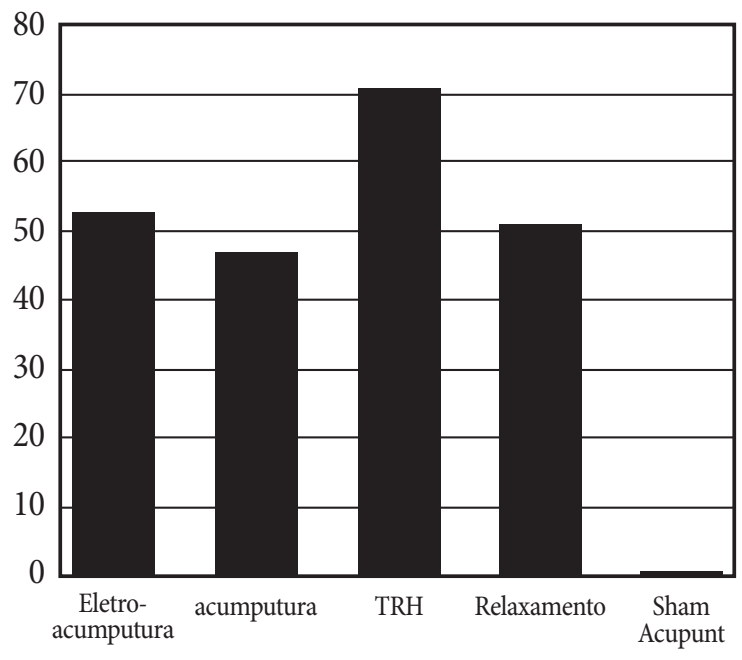

Quanto à média percentual apurada sobre ondas de calor, demonstraremos na Tabela 1 os resultados das técnicas analisadas nas variantes frequência e severidade.

Tabela 1. Basal X Pós Tratamento X Follow-up

\begin{tabular}{l|c|c}
\hline Técnica - para ondas de calor & Basal vs Pós Tratamento & Basal vs. Follow-up \\
\hline Eletroacupuntura quanto à frequência & 47,66 & 60,66 \\
\hline Eletroacupuntura quanto à severidade & 45,66 & 56,66 \\
\hline Acupuntura sistêmica quanto à frequência & 42,33 & 33,00 \\
\hline Acupuntura sistêmica quanto à severidade & 44,25 & 56,00 \\
\hline Sham - Acupuntura quanto à frequência & 35,00 & 31,50 \\
\hline Sham - Acupuntura quanto à severidade & 32,16 & 58,0 \\
\hline Relaxamento quanto à frequência & & \\
\hline Relaxamento quanto à severidade & 48,5 & \\
\hline Cuidados habituais quanto à frequência & 23,00 & 81,50 \\
\hline Cuidados habituais quanto à severidade & 28,00 & 89,66 \\
\hline Terapia de reposição hormonal quanto à frequência & 79,33 & 56,00 \\
\hline Terapia de reposição hormonal quanto à severidade & 79,50 & \\
\hline Terapia de reposição hormonal - placebo & 51,00 & \\
\hline
\end{tabular}

\section{TÉCNICA X PÓS TRATAMENTO}

A eletroacupuntura demonstrou uma diminuição média de $47 \%$ na variante frequência e $45 \%$ na variante severidade.

Com relação à acupuntura sistêmica, os números observados foram em torno de $42 \%$ e $44 \%$, nas variantes frequência e severidade, respectivamente. $\mathrm{O}$ que mostra que não há diferença significativa entre a eletroacupuntura e a acupuntura sistêmica nessa etapa do tratamento.

Cuidados habituais e sham-acupuntura foram as técnicas que menos produziram efetividade, em relação aos índices basais, mas, ainda assim produziram algum efeito. 
A TRH foi a técnica comparação que surtiu um efeito mais positivo para diminuição das ondas de calor, nas duas variantes; em torno de $79 \%$, tendo o placebo TRH 51\%.

\section{TÉCNICA X FOLLOW-UP}

Ao observar a técnica aplicada em longo prazo, os números se modificam significativamente em relação às técnicas; a TRH continua sendo a prática mais efetiva em longo prazo; $81 \%$ na variante frequência e $89 \%$ na variante severidade.

Analisando a eletroacupuntura e acupuntura sistêmica, diferentemente dos números apresentados após o tratamento, a EA aumenta sua eficácia em longo prazo nas duas variantes; frequência (60\%) e severidade (56\%), não ocorrendo o mesmo com a AC que só eleva sua média percentual na variante severidade (56\%).

Outro item que demonstrou alterações mais significativas em longo prazo foi a técnica comparativa relaxamento aplicado.

As demais técnicas, sham acupuntura, cuidados habituais não apresentaram valores expressivos, por não terem sido avaliadas no follow-up.

\section{TÉCNICA DA MTC X ONDAS DE CALOR X VARIANTES}

Comparando-se as variantes e as técnicas da MTC, os valores em relação às ondas de calor são similares (Ilustração no Gráfico 2).

Gráfico 2. Resultado $\mathrm{EA}^{*}$. $A C^{\star}$.Sham ${ }^{\star}$ - Ondas de calor x Frequência(F) x Severidade $(S)$

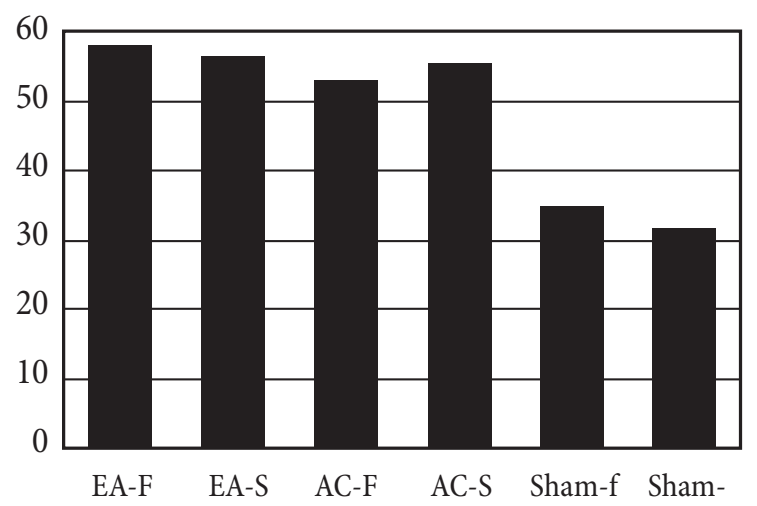

* EA- Eletroacupuntura AC - Acupuntura sistêmica - Sham -Acupuntura Simulada
Porém, ao analisar os trabalhos individualmente, é possível observar algumas particularidades: Frisk $^{13}$ demonstra em seu estudo que há um efeito positivo em torno de $56 \%$, tanto na frequência quanto na severidade para ondas de calor, no pós-tratamento, porém quando observado em longo prazo (follow-up) a eletroacupuntura com relação à frequência possui um efeito mais benéfico (78\%). Frisk utilizou-se da mesma técnica aplicada por Wyon $^{29}$, que não avaliou essa variante, portanto não existe um padrão comparativo com esse autor, mas, quando comparado a Huang $\mathrm{MI}^{15}$, que se utilizou de outra técnica, essa variante também produz valores significantes.

Ainda em relação à eletroacupuntura, Jue Zhou ${ }^{16}$ observou um número mais significativo em relação à severidade: $73 \%$ pós-tratamento e $69 \%$ no follow-up.

Em relação à acupuntura sistêmica, os números observados variaram muito - entre $38 \%$ e $56 \%$ apesar das demonstrações de eficácia do método, não podemos sugerir quais os melhores pontos para tratamento, porém a técnica aplicada nos estudos de Zaborowska $^{30}$ e Nedstrand e Wyon ${ }^{29}$, que fazem parte do mesmo grupo de pesquisa da Suécia, apresentaram valores mais expressivos, em torno de 51\%.

Sham acupuntura apresentou muita oscilação não podendo ser considerada uma terapia segura para diminuição de ondas de calor.

Quando observamos os números de Cohen et al ${ }^{9}$, valores expressivos na técnica placebo foi observada (76\%), porém devemos ressaltar que esse estudo em seu grupo comparativo(considerado nesse trabalho como placebo) utilizou-se de uma técnica comum da MTC; pontos de tonificação geral, para harmonização global, que permitem um efeito positivo nas pacientes avaliadas.

Cohen et al ${ }^{9}$ observaram ainda a aplicabilidade da acupuntura nos distúrbios do sono, mostrando diminuição significativa tanto no grupo experimental como no grupo controle (placebo);(29 - 13,0 e 22 - 11), respectivamente.

Jue Zhou et al ${ }^{16}$ também observaram mudanças nos níveis de FSH, LH e E2, sendo que, após o tratamento, os níveis de FSH diminuíram signifi- 
cativamente e o de E2 aumentaram em ambos os grupos(acupuntura e TRH), porém o LH somente diminuiu significativamente no grupo TSH.

Borud, EK et al ${ }^{10}$ analisaram sintomas vasomotores, problemas de sono e problemas somáticos através do WHQ. Os diagnósticos foram efetuados através dos princípios da MTC para diagnosticar síndromes primárias e sintomas da menopausa.

Optamos em analisar esse estudo separadamente, pois o objetivo principal foi verificar quais eram os padrões sindrômicos, segundo a MTC, dominantes nas mulheres que fazem parte do Grupo de Climatério.

As síndromes observadas dentro da MTC para esse estudo foram: Deficiência de Yin de Rim com vazio de coração, Deficiência de Yang de Rim por vazio de frio (falso Yang), Deficiência de Yin e Yang de Rim, Deficiência de Yin de Rim e Fígado, com Yang de Fígado em ascensão, Rim e Coração não se harmonizam

A síndrome mais encontrada entre mulheres no climatério foi Deficiência de Yin de Rim; 50\%.

\section{DISCUSSÃO}

Su Wen, citado por Auteroche ${ }^{4}$, diz que "[...] as alterações femininas ocorrem de 7 em 7 anos", e“[...] aos 49 anos o Ren Mai é flácido, o Tai Chong Mai atrofiado, o Tian Gui esgotado, as vias subterrâneas cortadas e a infecundidade resulta desse esgotamento do corpo". Essa passagem de Su Wen põe em evidência a importância do estado do Qi (energia) do Rim e a dos Vasos Chong Mai e Ren Mai na atividade fisiológica da mulher.

A síndrome climatérica ocorre quando o $Q i$ dos Rins está gradualmente enfraquecido e Chong Mai e Ren Mai estão vazios e o Tian Gui esgotado; então as menstruações param progressivamente, nessa época da vida pode sobrepor-se uma fraqueza corporal ou um distúrbio psíquico proveniente dos sete sentimentos que são o reflexo da perda de equilíbrio do Yin e do Yang.

Através dessa citação é possível, claramente, observar a correlação entre a MTC e a medicina ocidental em relação às alterações físicas e emocionais da mulher na sua fase de transição menopausal.
Nessa passagem, a data próxima da menopausa é 49 anos, como já observado anteriormente. Su Wen, então, registra os fenômenos da transição da vida da mulher, no qual cita a falência dos ovários, a cessação da menstruação, as alterações dos sentimentos, tudo isso relacionado aos processos naturais do envelhecimento, neste caso, caracterizado pela deficiência de Qi (energia) do Rim.É importante salientar que na MTC o Rim está relacionado com a essência vital/genética.

Atualmente existem algumas controvérsias na área da saúde quanto à prática da acupuntura; se energética ou neurofisiológica. Nos estudos observados não há citações explícitas quanto ao método utilizado, apenas fazem citações quanto à importância em alcançar-se o chamado "De Que" (sensação de queimação, ardor, calor) ao ser pontuado.

Nota-se que, apesar da escassez dos estudos na área de particularidades femininas, a prática clínica nos tratamentos de sintomas do climatério através da acupuntura não apresenta contra indicação e não apresenta efeitos colaterais nos estudos analisados, sendo uma alternativa sem riscos para as mulheres.

As maiores questões, porém, são abordadas em relação à população observada e ao método placebo.

Nos estudos analisados, a população média em estudo ficou em torno de 54 mulheres, e os métodos de sham-acupuntura adotados não são aceitos na comunidade científica, até a presente data.

Observou-se que Jue Zhou ${ }^{16}$ foi o único autor que propôs análises observando os níveis de E2/LH/ FSH como parâmetro para observar o mecanismo de ação da acupuntura, talvez essa seja uma forma de monitorar as alterações orgânicas que ocorrem durante e depois das sessões.

Mesmo não havendo clareza em muitos aspectos, os números observados possuem uma inclinação favorável para o tratamento de eletroacupuntura e acupuntura sistêmica para sintomas da menopausa.

Acredita-se que o mecanismo de ação da acupuntura possa influenciar sobre a produção e secreção de hormônios neurotransmissores ou moduladores do HPOA. Talvez esse seja o caminho mais promissor a seguir. 
Zhou, Ch et al, citado por Bo-Yin $\mathrm{Chen}^{27}$ notaram algumas alterações nos níveis de sangue de $\mathrm{LH}$, FSH e E2 e prolactina nas pacientes femininas. São resultados significativos, mas insuficientes para explicar o mecanismo da acupuntura na regulação da função do HPOA.

Nesse mesmo estudo, um grupo de mulheres e um grupo de ratas foram observados para cronicidade anovulatória e os resultados após a EA foram aumento dos níveis de E2 e diminuição das taxas de FSH.

O autor tem efetuado trabalhos desde 1985, observando o efeito da EA na indução ovulatória. EA sugere que poderia promover a atividade dos mecanismos compensatórios para elevar o nível subnormal de EA induzida pela ovariectomia. O autor tem efetuado esses estudos em ratas ovariectomizadas.

A acupuntura é uma prática também utilizada em animais, e, nesse caso, os pontos de acupuntura utilizados foram similares ao utilizados nos tratamentos em mulheres no climatério; Sanyinjiao (BP6), Guanyuan (VC4), Waiguan (CS5), Zhongji (VC3), Ponto Extra Huantuo Jiaji (Extra 15), Ponto
Extra Zigong do Abdômen (Extra 22), com EA de na frequência de $3 \mathrm{~Hz}$.

\section{CONCLUSÃO}

Nesta proposta, foi possível observar que a acupuntura e a eletroacupuntura demonstram ser uma prática positiva para amenizar os afrontamentos do climatério, principalmente em mulheres que possuem contra indicação relativa ou absoluta para a TRH.

Participações mais efetivas dos profissionais da área colaborariam no sentido de elucidar as dúvidas persistentes; resultados práticos da clínica diária seriam suficientes para caracterizar a acupuntura como método alternativo seguro.

Estudos mais amplos, envolvendo números maiores de participantes, descoberta e aplicação de um método sham-acupuntura aceito na comunidade científica, descoberta do mecanismo de ação da acupuntura sobre as ondas de calor, entre outros fatos, colaborariam para esclarecer as dúvidas ainda remanescentes.

\section{CONFLITOS DE INTERESSE}

Declarou não haver

\section{FONTES DE FINANCIAMENTO}

Nenhuma

\section{AUTORIA}

Todas as autoras participaram da concepção, busca de materiais, redação e revisão do artigo.

\section{REFERÊNCIAS}

1 - Aldrigui JM. Climatério. ARS CURANDI, 1994, 9: 5.

2 - Aldrighi JM. Autoconcepção no climatério. Feminina. 1992; 20:274.

3 - Auteroche B, Navailh P. Acupuntura em Ginecologia e Obstetrícia. Andrei Editora, 1987,pg. 20; 127-135.

4 - Avis NE, Legault C, Coeytaux RR, Pian-Smith M, Shifren JL, Chen W, Valaskatgis P. A randomized, controlled pilot study of acupuncture treatment for menopausal hot flashes. Menopause; 2008. Nov-Dec; 15(6):1070-8.

5 - Berek JS. Berek \& Novak’s Gynecology. Philadelphia: Lippincott Williams \& Wilkins, 2007.

6 - Berni N, Luz MH, Kohlraush SC. Conhecimento, percepção e assistência à saúde da mulher no climatério. Revista Brasileira de Enfermagem, 2007;60(3):299-306.

7 - Borud Einar Kristian, Teije Alrak, Adrian White, Sameline Grimsgaard. The acupuncture treatment for postmenopausal hot flushes(Acuflash) study: traditional Chinese Medicine diagnoses and acupuncture points used, and their relation to the treatment response. Acupuncture in Medicine, 2009, v.27, 101-108.
8 - Bo-Yin Chen or Chen BY. Acupuncture normalizes dysfunction of hypothalamic-pituitary-ovarian axis. Acupunct Eletrother Res, 1997; 22:97-108.

9 - Cohen SM, Rosseau ME, Carey BL. Can acupuncture ease the symptoms of menopause? Holistic Nursing Practice, 2003- NovDec: 17(6):295-9.

10 - Deng G, Vickers A, Yeung S, D 'Andrea GM, Xiao H, Heerdt AS, Sugarman S, Troso-Sandoval T, Seidman AD, Hudis CA, Cassileth B. Randomized, controlled trial of acupuncture for the treatment of hot flashes in breast cancer patients. Journal of Clinical Oncology, 2007 Dec 10; 25(35): 5584-90.

11 - Elias S, Dardes RCM, Kemp C, et al. Estudo piloto dos efeitos da terapia hormonal sobre o tecido mamário normal de mulheres após a menopausa. Revista Brasileira de Ginecologia e Obstetrícia, v.28, n.11. Rio de Janeiro. Nov. 2006.

12 - Freitas KM et al. Mulheres vivenciando o climatério. Acta Scientiarum Health Sciences. Maringá, v. 26, no. 1, p. 121-128, 2004. 
13 - Frisk J, Carlhäll S, Källström AC, Lindh-Astrand L, Malmström A, Hammar M. Long-term follow-up of acupuncture and hormone therapy on hot flushes in women with breast cancer: a prospective, randomized, controlled multicenter trial. Climacteric; 2008 Apr; 11(2):166-74.

14 - Holmberg L, Anderson H. HABITS(hormonal replacement therapy after breast câncer - is it safe?) a randomized comparasion: trial stapped. The Lancet, 2004; 7:453-5.

15 - Huang MI, Nir Y, Chen B,Schnyer R, Mamber R. A Randomized controlled pilot and sleep quality. Fertil Steril, 2006 Sep; 86(3): 700-10.

16 - Jue Zhou et. al. Acupuncture and Auricular acupressure in relieving menopausal hot flashes of bilaterally ovariectomized Chinese women: A Randomized controlled Trial.). EvidenceBased Complementary and Alternative Medicine, 2009, Feb 2, 1-8.

17 - Kronenberg F. Hot Flashes: epidemiology and physiology. Annals of the New York Academy of Sciences, 1990: 592:52-86.

18 - Kroenberg F. Hot flashes: Phenomenology, quality of life, and search for treatment options. Experimental Gerontology, 1994; 29, 771-90.

19 - Kronenberg F, Fugh-Berman, A. Complementary and alternative Medicine for Menopausal Symptoms: A Review of Randomized, Controlled Trials. Annals of Internal Medicine, 2002; 137:805-813.

20 - McKinlay SM, Jefferys M. The menopausal syndrome. Br J Prev. Soc. Med. 1974; 28(2):108-15

21 - Nedstrand E, Wijma K, Wyon Y, Hammar M. Vasomotor symptoms decrease in women with breast cancer randomized to treatment with applied relaxation or electro-acupuncture: a preliminary study. Climacteric: 2005 Sep; 8(3):243-50.
22 - Nir Y, Huang MI, Schnyer R, Chen B, Mamber R. Acupuncture for postmenopausal hot flashes. Maturitas; 2007 Apr 20; 56(4): 383-95.

23 - Shanafelt TD, Barton DL, Adjei AA, Loprinzi Cl. Pathophysiology and treatment of hot flashes. Mayo Clinic Proceedings, 2002; 77(11):1207-18.

24 - Stearns V, Ullmer L, et al. Hot flushes. The Lancet, 2002; 200:360(9348):1851-1861.

25 - Vincent A, Barton DL, Mandrekar JN, Cha SS, Zais T, WahnerRoedler DL, Keppler MA, Kreitzer MJ, Loprinzi C. Acupuncutre for hot flashes: a randomized, sham-controlled clinical study. Menopause, 2007 Jan-Feb; 14(1): 45-52.

26 - Woods NF, et al. Symptoms during the menopausal transition and early postmenopausae and their relation to endocrine levels over time: observations from the Seattle Midlife Women's Health Study. Journal of Women's Health, 2007; 16:667-77.

27 - World Health Organization (WHO). Guidelines on Basic Training and Safety In Acupuncture. Geneva: WHO Publications, 1999.

28 - Wyon Y, Lindgren R, Lundeberg T, Hammar M. Effects of acupuncture on climacteric vasomotor symptoms, quality of life and urinary excretion of neuropeptides among postmenopausal women. Menopause, 1995; 2-3-12.

29 - Wyon Y, Wijma K, Nedstrand E, Hammar M. A comparison of acupuncture and oral estradiol treatment of vasomotor symptoms in postmenopausal women. Climacteric: 2004 Jun: 7(2): 153:164.

30 - Zaborowska E, Brynhildsen J, Damberg S, Fredriksson M, Lindh-Astrand L, Nedstrand E, Wyon Y, Hammar M. Effects of acupuncture, applied relaxation, estrogens and placebo on hot flushes in postmenopausal women: an analysis of two prospective, parallel, randomized studies. Climacteric; 2007, Feb; 10(1):38-45. 